\title{
LITIASIS RENAL EN PACIENTE CON ENFERMEDAD DE CROHN: REPORTE DE CASO
}

\section{RENAL LITHIASIS IN PATIENT WITH CROHN'S DISEASE: CASE REPORT}

Alexander Oswaldo Ojeda-Crespo ${ }^{1}$, Alexander Xavier Ojeda-Cedillo², Andrés Eduardo Ojeda-Cedillo², Peter Oswaldo Ojeda-Cedillo², Edgardo Mengual ${ }^{3}$.

\section{RESUMEN}

Caso de litiasis renal por oxalato de calcio asociado a enfermedad de Crohn de reciente debut en paciente masculino 43 años proveniente del Canton las Piñas, Proviancia El Oro en Ecuador, sin antecedentes patológicos en relación a su enfermedad. En enero 2020 acude a consulta particular con dolor en fosa iliaca derecha, fiebre, fatiga, hiporexia, pérdida de peso, tenesmo y diarrea. Gastroenterólogo observó colon derecho con úlceras, áreas de mucosa infiltrada,y el estudio anatomopatológico identifica ulceraciones en subserosa, abscesos, infiltrado inflamatorio mixto, granulomas epitelioides con células gigantes multinucleadas. Previo consentimiento se realiza ecosonografía, detectándose un cálculo renal derecho de $5 \mathrm{~mm}$ de tamaño y otro de $4 \mathrm{~mm}$ en el izquierdo. El análisis de orina reporta aciduria, cristales oxalato de calcio y la radiografía simple demostró sombra de los cálculos. El estudio metabólico la I,25 dihidroxivitamina $D$ y la hormona paratiroidea resultó negativo. La evolución fue favorable y continua en control periódico que de ameritar se ordenaría tomografía helicoidal sin contraste o urograma excretorio.

\section{ABSTRACT}

We present a case of kidney stones due to calcium oxalate stones associated with Crohn's disease of recent debut in a 43-year-old male patient from the Piñas canton-El Oro Province-Ecuador with no personal and family pathological history in relation to his disease and that in January 2020 he went to a private medical consultation for presenting colic-type abdominal pain located in the right iliac fossa, fever, fatigue, hyporexia, weight loss, tenesmus and sometimes diarrhea. The gastroenterologist observed: right colon with ulcers and infiltrated mucosa areas; samples are taken for histopathological study. Anatomopathology It was identified ulcerations in subserosa, abscesses, mixed inflammatory infiltrate, epithelioid granulomas with multinucleated giant cells diagnosed with Crohn's disease. With the patient's consent, an echo-sonographic study was made, detecting a right kidney stone $5 \mathrm{~mm}$ in size and $4 \mathrm{~mm}$ in the left. Urinalysis showed aciduria, calcium oxalate crystals, and plain abdominal radiography showed shadow of the stones. In the metabolic study the I,25 dihydroxyvitamin D and parathyroid hormone was negative. The evolution was favorable and he continuous in periodic control that, if it's required, would be ordered a helical computed tomography without contrast or excretory urogram.

\section{INTRODUCCIÓN}

a nefrolitiasis es una patología común

Ly aproximadamente el 5 a 12\% de la población va a desarrollar cálculos antes de los 70 años. La enfermedad de Crohn (EC), colitis ulcerativa (CU) y la enfermedad inflamatoria no clasificada (EllnC) son enfermedades crónicas del intestino, de carácter inflamatorio, conocidas en conjunto como enfermedad inflamatoria intestinal (Ell), sin etiología definida, con distintas características clínicas, patológicas, endoscópicas y radiológicas que ocasionan efectos extraintestinales como la nefrolitiasis'.

La mayoría de los pacientes con EC presentan un elevado riesgo de padecer litiasis renal de oxalato cálcico,que dicho riesgo es multifactorial, de modo que cada paciente puede presentar varias anomalías metabólicas litogénicas las cuales son más evidentes durante los episodios de actividad de la enfermedad ${ }^{2}$. Existen factores desencadenantes como las alteraciones físicoquímicas, desequilibrio endocrino-metabólico y alteraciones anatómicas o hidrodinámicas. Un solo paciente regularmente tiene varios trastornos metabólicos que son más evidentes en el episodio activo de la enfermedad ${ }^{3}$.

Se ha descrito mayor frecuencia de litiasis renal en aquellos pacientes con más de 40 años al diagnóstico de la enfermedad intestinal, que
${ }^{1}$ Médico, Docente Investigador, Universidad Técnica de Machala, El Oro, Ecuador ${ }^{2}$ Médico posgradista Universidad del Zulia, Maracaibo. Venezuela ${ }^{3}$ Médico especialista Gastroenterólogo Universidad del Zulia, Maracaibo, Venezuela

Correspondencia a:

Alexander Oswaldo OjedaCrespo.

Correo electrónico: aojeda@ utmachala.edu.ec

ORCID: http://orcid.org/00000003-2657-1736.

Telefono: 0991595724

Palabras clave: Enfermedad de Crohn, litiasis, intestino grueso, colonoscopía

Keywords: Crohn's disease, lithiasis, large intestine, colonoscopy

Procedencia y arbitraje: no comisionado, sometido a arbitraje externo.

Recibido para publicación: 14 de abril 2020 Aceptado para publicación: 3 de julio 2020

Citar como:

Ojeda-Crespo AO, OjedaCedillo AX, Ojeda-Cedillo AE, Ojeda-Cedillo PO, Mengual E. Litiasis renal en paciente con enfermedad de crohn: Reporte de caso. Rev Cient Cienc Med. 2020; 23(1): 97-101 
precisamente representa el grupo de edad, entre 30 y 50 añosl; optando por investigar a los pacientes con enfermedad de Crohn, el caso presente se encuentra en ese rango de edad $^{4}$. Mediante la realización de exámenes complementarios y el trabajo multidisciplinario con las diferentes especialidades se establece su relación con la litiasis renal. En Ecuador no existe estudio alguno sobre esta complicación extra intestinal.

\section{PRESENTACIÓN DEL CASO}

Es el caso de un paciente del género masculino de 43 años de edad procedente del cantón Piñas-Provincia de El Oro-Ecuador y que en enero 2020 acude a consulta médica particular por presentar dolor abdominal tipo cólico localizado en fosa iliaca derecha continuo y poco irradiado, fiebre,fatiga, hiporexia, pérdida de peso desde el inicio de su enfermedad, dolor con la defecación, tenesmo y en ocasiones diarrea. El paciente refiere que este cuadro clínico se ha repetido en cuatro ocasiones anteriores. Se le realizó tratamiento ambulatorio con un diagnóstico previo de infección intestinal y amebiasis. Sin embargo la signo-sintomatología persistía y regresa a consulta, siendo referido al gastroenterólogo quien le practica colonoscopia, observando: colon derecho con úlceras y áreas de mucosa infiltrada, donde se toman muestras para estudio histopatológico (Ver figura 1). Se ordena luego de este procedimiento una TAC abdominal, donde se visualiza engrosamiento de íleon distal y colon derecho con líquido libre laminar en cavidad abdominal. El estudio anatomopatológico identificó ulceraciones que comprometen hasta la subserosa, absceso en las criptas e infiltrado inflamatorio mixto hasta el tejido adiposo (Ver figura 2). Se identifican granulomas epitelioides con células gigantes multinucleadas en todo el espesor de la pared, estableciendo el diagnóstico de Enfermedad de Crohn (Ver figura 3).

Ante los hallazgos de la colonoscopiaTAC y estudios anatomopatológicos el gastroenterólogo decide comenzar tratamiento con corticoides parenteral, azatioprina y mesalazina oral. Conociendo del caso el radiólogo procede previo el consentimiento del paciente a realizar un estudio ecosonográfico, detectándose un cálculo renal derecho de 5

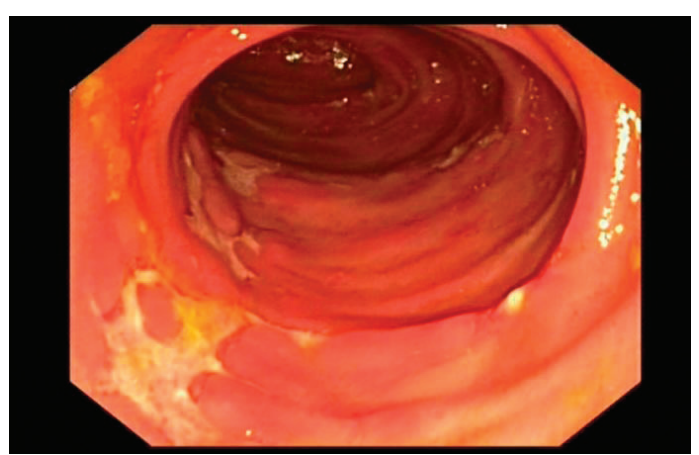

Figura 1: Ulcera con bordes irregulares agrietado

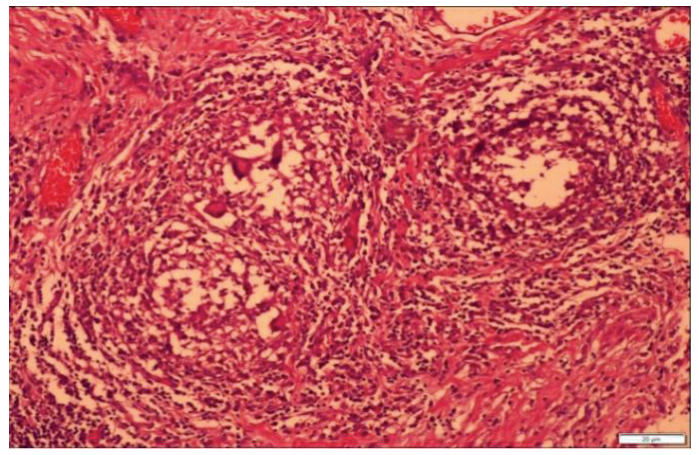

Figura 2: tinción $\mathbf{H \& E}, \mathbf{5 X}$ : Los cortes histológicos corresponden a pared de intestino, la mucosa conformada por glándulas y criptas revestidas por epitelio cilíndrico simple bien diferenciado con severa respuesta inflamatoria mixta linfocitos, células plasmáticas y polimorfonucleares, en áreas se observa absceso en las criptas, focalmente se identifica granulomas epitelioides con células gigantes multinucleadas en todo el espesor de la pared. Laboratorio de patología Dra. Sylvana Cuenca Buele. Nro Archivo: 018-20

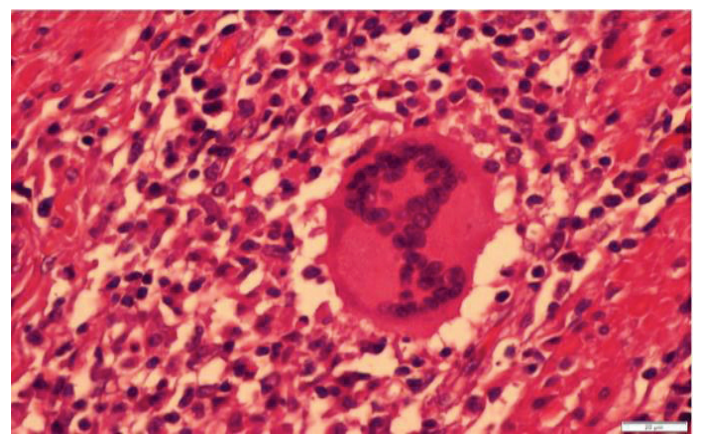

Figura 3: H\&E-40X: Célula gigante multinucleada en uno de los granulomas. Laboratorio de patología Dra. Sylvana Cuenca Buele. Nro. Archivo: 018-20

$\mathrm{mm}$ de tamaño y otro de $4 \mathrm{~mm}$ en el izquierdo (Ver figura 4).

Los resultados del análisis de orina mostraron aciduria, cristales de oxalato de calcio y la radiografía simple de abdomen mostró sombra de los cálculos. 


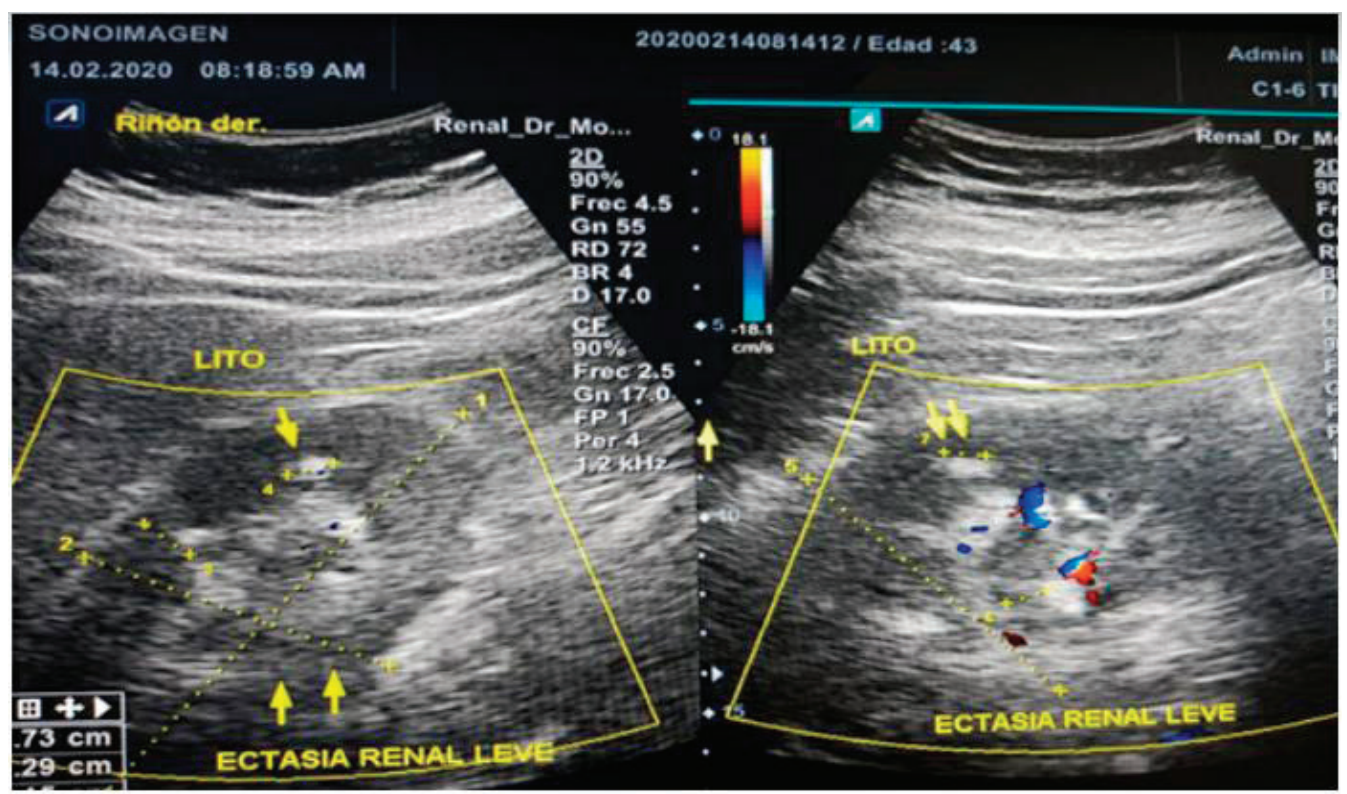

Figura 4: Ecografía con presencia de litiasis renal bilateral

Las recomendaciones dietéticas son generales para cualquier tipo de litiasis, y varían en función de la anomalía metabólica. La ingesta elevada de agua (2.000-3.000 $\mathrm{ml} / 1,73 \mathrm{~m} 2$ ) ha mostrado ser beneficiosa $y$ coste efectivo, con reducción de un $56 \%$ del riesgo de recurrencia. Evitar restricción severa dietética de calcio (ingesta recomendada 1000 - 1200mg/día) para quelar el oxalato intestinal. Evitar alimentos ricos en oxalato como nueces chocolate, espinacas, té, remolacha, judías, patatas, etc. Además, se le prescribió citrato de potasio ( $1 \mathrm{mg} / \mathrm{kg}$ de peso) en tabletas.

El paciente se mantiene con la medicación del gastroenterólogo antes mencionada y se encuentra estable con evolución favorable. Se programaron controles periódicos.

\section{DISCUSIÓN}

La nefrolitiasis es la complicación renal dentro de las manifestaciones extraintestinales de la Enfermedad de Crohn, asociándose el Oxalato de calcio como la principal fuente de composición de estos cálculos, cursando de forma característica con hiperoxaluria secundaria 5 .

En Latinoamérica son escasos los estudios realizados, identificando así en Sao Paulo, Brasil (2013) un análisis poblacional de 93 pacientes con EC, con edad media de 42 años, de los cuales 28 pacientes (38\%) presentaron nefrolitiasis, siendo mayor en pacientes con enfermedad ileocolónica. Estos datos son semejantes al estudio antes mencionado, sin embargo el antecedente de cirugía previa fue insuficiente para describir su relación como factor de riesgo ${ }^{6}$.

Mientras que, en Corea, en el año 2015 se describe una incidencia de 18 pacientes $(4,7 \%)$ con nefrolitiasis y una incidencia anual de $(0,7 \%)$, de una población total de 387 pacientes con diagnóstico de EC, confirmado con estudios de imagen ${ }^{7}$.

Datos similares se obtuvieron en un análisis prospectivo realizado en Suiza (2017), con un grupo poblacional de 1333 pacientes con EC, de ellos 61 (4,6\%) desarrollaron cálculos renales, asociando significativamente algunos factores como: la duración y actividad de la enfermedad, la cirugía intestinal, el sexo masculino y sedentarismo ${ }^{8}$.

Al igual que África (2013), se realizó una investigación retrospectiva durante 10 años, de 184 pacientes tunecinos con EC, de los cuales solamente 3 pacientes presentaron nefrolitiasis, de composición oxalocálcica, $(1,63 \%)$ todos localizados en el lado derecho y mujeres con edad promedio de 34 años, el diagnóstico se confirmó con ecografía y tomografía computarizada9-10. Estos datos obtenidos de Asia (Corea), Europa (Suiza) y de África son ampliamente menores a los descritos en la literatura, ligados posiblemente a factores genéticos o ambientales propios de 
estas poblaciones ${ }^{11}$.

El paciente no presenta antecedentes personales de gota, hiperparatiroidismo primario, acidosis tubular renal, fracturas óseas patológicas ni otro factor de riesgo significativo. En el estudio metabólico para determinar la 1,25 dihidroxivitamina $D$ y la hormona paratiroidea fue negativo. Además, se le realizó dos muestras de orina de 24 horas para valorar: volumen, $\mathrm{pH}$, creatinina, calcio, fosfato, ácido úrico, oxalato, citrato, sodio, potasio, magnesio y amonio. Deben recogerse dos muestras, puesto que existe gran variabilidad en los resultados de diferentes muestras ${ }^{12}$.

Cuando el tratamiento conservador fracasa, el paciente debe ser derivado al urólogo para su tratamiento definitivo como lo es la litotricia extracorpórea por ondas de choque que es usado habitualmente por ser menos invasivo y no requerir ingreso, pero tiene algunas limitaciones, como un alto porcentaje de retratamientos (entre el 4 y el 50\%). Esta, está indicada como tratamiento de primera línea en Litiasis renales $\leq 20 \mathrm{~mm}^{13}$.

La evolución fue favorable y continua en control periódico que de ameritar se ordenaría Tomografía computarizada helicoidal sin medio de contraste o urograma excretorio. Dentro de las recomendaciones dietarías se estableció la ingesta de calcio normal (1000-1200 mg/día de calcio elemental); restricción moderada de sal, proteínas de origen animal, ingesta adecuada de agua, frutas, verduras y cereales integrales ${ }^{14}$.

\section{CONCLUSIÓN}

Con el presente caso clínico, se ha demostrado que el riesgo para desarrollar litiasis renal en pacientes que cursan con Enfermedad de Crohn a lo largo de su vida, es factible y se puede correlacionar con los datos descritos en la literatura, con una prevalencia estimada entre $12 \%-28 \%$, además es mayor cuando la enfermedad es ileocolónica (9\%$17 \%)$ que ileal (6\%-8\%) o solo colónica (3\%-
$5 \%)$. Sin embargo, son limitados los estudios realizados en la última década.

Por esta razón la detección, prevención y el tratamiento de cálculos en pacientes con enfermedades del colon son aspectos importantes en su manejo clínico, tanto para la prevenir el retraso en el diagnóstico así como evitar secuelas graves asociadas a la nefrolitiasis entre ellas, la pérdida de la función renal. Además del tratamiento estos pacientes deben recibir seguimiento clínico, con el objetivo de prevenir la tasa de recurrencias, preservar la función renal, reducir la morbilidad y sobre todo mejorar la calidad de vida.

Se requiere especial atención en estos pacientes para prevenir la reincidencia o disminuir los factores de riesgo que influyen en la formación de nefrolitiasis oxalocálcica asociada a la Enfermedad de Crohn. Creemos que este caso es útil y actualizado en nuestro país, ya que no existen datos estadísticos reportados hasta la presente fecha y en Latinoamérica son escasos.

Conflicto de intereses. - Los autores declaran no tener ningún conflicto de intereses con el hospital y que se tomó el consentimiento informado al paciente con derecho a la privacidad. Declaran que para esta investigación no se han realizado experimentos en seres humanos, no aparecen datos de pacientes y fue financiado en su totalidad por los autores. 


\section{REFERENCIAS}

1.- Corica D, Romano C, Renal Involvement in Inflammatory Bowel Diseases, Journal of Crohn's and Colitis, Volume 10, Issue 2, February 2016, Pages 226-235. Disponible en: https://doi.org/10.1093/ ecco-jcc/jjv138

2.- Ganji-Arjenaki M, Nasri H, Rafieian-Kopaei $M$. Nephrolithiasis as a common urinary system manifestation of inflammatory bowel diseases; a clinical review and meta-analysis. J Nephropathol. 2017;6(3):264-269. Disponible en: doi:10.15171/ jnp.2017.42

3.-Cury, Didia, et al. "Nephrolithiasis in Patients with Inflammatory Bowel Disease in the Community». International Journal of Nephrology and Renovascular Disease, julio de 2013, p. 139. Disponible en: DOl.org (Crossref), doi:10.2147/ IJNRD.S45466.

4.-Gaspar SR. Mendonca T, Oliveira P, Oliveira T, Dias J, López T. Urolithiasis and Crohn disease. Urol, Ann (internet).2016; 8:297-304. Disponible en: http:// www.urologyannals.com/article.asp?issn=09747796; year=2016; volume=8;issue=3; spage=297; epa ge=304; aulast=Gaspar

5.- Fagagnini S, Heinrich $H$, Rossel JB. Risk factors for gallstones and kidney stones in a cohort of patients with inflammatory bowel diseases. PLoS One. 2017;12(10):e0185193. Published 2017 Oct 12. doi: 10.1371/journal.pone.0185193. Disponible en: https://doi.org/10.1371/journal.pone.0185193

6.-Mendoza JL Lana R Taxonera do Alba do Izquierdo $S$ Díaz-Rubio. Manifestaciones extraintestinales en la enfermedad inflamatoria intestinal: diferencias entre la enfermedad de Crohn y la colitis ulcerosa. Med Clin (Barc) 2015; 125:297 - 300. Disponible en: https://doi:10.1157/13078423

7.- Mukewar S, Hall P, Lashner BA, Lopez R, Kiran RP, Shen B. Risk factors for nephrolithiasis in patients with ileal pouches. J Crohn's Colitis [Internet].
2013;7(1):70-8. Available from: http://dx.doi. org/10.1016/j.crohns.2012.05.006

8.- Burisch J, Jess T, Martinato M, Lakatos P. The burden of inflammatory bowel disease in Europe. World Journal Of Gastroenterology. 2013. 7: 322-337. Disponible en: http://dx.doi: 10.1016/j. crohns.2013.01.010

9.- Kim MJ, Woo S-Y, Kim ER, Hong SN, Chang DK, Rhee $P-L$, et al. Incidence and Risk Factors for Urolithiasis in Patients with Crohn's Disease. Urol Int. 2015;95(3):314-9. Disponible en: https://www. ncbi.nlm.nih.gov/pmc/articles/PMC4155345/

10.- Josephine M. Ambruzs, Christopher P. Larsen. Renal Manifestations of Inflammatory Bowel Disease.Rheum Dis Clin North Am. 2018 Nov; 44(4): 699-714. Disponible en: https://doi: 10.1016/j. rdc.2018.06.007

11.- Instituto Nacional de Estadísticas y Censos INEC. Estructura de la población El Oro 2018. Recuperado de: http://www.ecuadorencifras.gob.ec/wpcontent/descargas/Manu-lateral/Resultadosprovinciales/el_oro.pdf

12.-Sorokin I, Mamoulakis C, Miyazawa K, Rodgers A, Talati J, Lotan Y. Epidemiology of stone disease across the world. World J Urol. 2017 Sep;35(9):13011320. Disponible en: Doi: 10.1016/j.ajur.2018.08.007

13.-Igor Sorokin a, Margaret S. Pearle. Medical therapy for nephrolithiasis: State of the art. Asian Journal of Urology (2018) 5, 243-55. Disponible en: https://www.ncbi.nlm.nih.gov/pmc/articles/ PMC6197179/

14.-DiBianco JM, Jarrett TW, Mufarrij P. Metabolic syndrome and nephrolithiasis risk: should the medical management of nephrolithiasis include the treatment of metabolic syndrome? Rev Urol 2015;17:117-28. Disponible en: https://www.ncbi. nlm.nih.gov/pmc/articles/PMC4633655/ 